\title{
Local Approximate Forward Attractors of Nonautonomous Dynamical Systems
}

\author{
Ailing Qi, Xuewei Ju* \\ Department of Mathematics, Civil Aviation University of China, Tianjin, China \\ Email address: \\ alqi@ cauc.edu.cn (Ailing Qi), juxuewei@tju.edu.cn (Xuewei Ju) \\ ${ }^{*}$ Corresponding author
}

\section{To cite this article:}

Ailing Qi, Xuewei Ju. Local Approximate Forward Attractors of Nonautonomous Dynamical Systems. American Journal of Applied Mathematics. Vol. 8, No. 5, 2020, pp. 278-283. doi: 10.11648/j.ajam.20200805.16

Received: November 6, 2019; Accepted: December 20, 2019; Published: September 18, 2020

\begin{abstract}
Pullback dynamics of nonautonomous dynamical systems has been considerably developed. However, it is still a tough job to study forward dynamics of nonautonomous dynamical systems, since forward attractors were only obtained in some particular cases. In the paper, under some reasonable conditions, it is shown that closing to a local pullback attractor, there is an approximate forward attractor. Specifically, let $\phi$ be a cocycle semiflow on a Banach space $X$ with driving system $\theta$ on a base space $P$. Suppose that the base space $P$ is compact and $\phi$ is uniformly asymptotically compact. Let $A(\cdot)$ be a local pullback attractor with $\bigcup_{p \in P} A(p)$ being compact. We prove that every $\varepsilon$-extended neighborhood $A^{\varepsilon}(\cdot)$ of $A(\cdot)$ will forward attract every bounded set $B(\cdot)$ that is pullback attracted by $A(\cdot)$. We then call $A^{\varepsilon}(\cdot)$ an approximate forward attractor of $\phi$.
\end{abstract}

Keywords: Nonautonomous Dynamical Systems, Pullback Attractors, Approximate Forward Attractors

\section{Introduction}

The dynamics of nonautonomous dynamical systems is very complicated. As matter of fact, one can define two different types of attractors for nonautonomous dynamical systems: pullback attractors and forward attractors, corresponding to pullback attraction and forward attraction, respectively. A (local) pullback (resp. forward) attractor we talk about here is a compact, invariant set that pullback (resp. forward) attracts a neighborhood of itself. The existence of the former one can be obtained under quite standard hypotheses which are similar to those to guarantee the existence of attractors for autonomous systems; see e.g. [4, 9, 17]. However, the notion of forward attractors sounds to be more suitable to describe how nonautonomous systems evolve in the future. Unfortunately, the existence of forward attractors remains open problems except in some particular nonautonomous cases such as the asymptotically autonomous and the periodic ones, and are still under investigations; see e.g. Cheban et. al [2], Wang et. al [18]), Kloeden [12] and Carvalho et. al [15, pp. 595]. Therefore, it is still a tough job to study forward dynamics of nonautonomous systems.

Our framework in the paper is to treat a nonautonomous system as a cocycle semiflow over a suitable base space rather than a process. Specifically, let $\phi$ be a cocycle semiflow on a Banach space $X$ with driving system $\theta$ on a base space $P$. One of the advantage of the cocycle semiflows framework is that in many cases the base spaces are compact, while the default base space $\mathbb{R}$ (real number set) for processes is unbounded. Based on the compactness of the base spaces, the pullback attraction of cocycle semiflow and (forward) attraction of the associated autonomous semiflow is shown to be equivalent. This allows us to study the forward dynamics of nonautonomous systems through their associated autonomous semiflows. Suppose throughout the paper that the base space $P$ is compact. If $\phi$ is uniformly asymptotically compact and has a global pullback attractor $\mathscr{A}(\cdot)$, it was shown in Ju et al. [10] that every $\varepsilon$-extended neighborhood $\mathscr{A}^{\varepsilon}(\cdot)(\varepsilon>0)$ of $\mathscr{A}(\cdot)$, called a global approximate forward attractor, has forward attraction. It will forward attract any bounded set $B$ in the phase space $X$, that is, $\lim _{t \rightarrow \infty} H_{X}\left(\phi(t, p) B, \mathscr{A}^{\varepsilon}\left(\theta_{t} p\right)\right)=$ 0 , where $H_{X}(\cdot, \cdot)$ denotes the Hausdorff semidistance in $X$. In this paper, we extend the result in [10] to local case and get local approximate forward attractors. In fact, utilizing a very different method, we obtain a stronger result, obtaining approximate forward attracting sets associated with pullback attracting sets. Specifically, suppose $K(\cdot)$ and 
$B(\cdot)$ are two nonautonomous sets with $\overline{\bigcup_{p \in P} K(p)}$ being compact. If $K(\cdot)$ pullback attracts $B(\cdot)$, then every $\varepsilon$-extended neighborhood $K^{\varepsilon}(\cdot)$ of $K(\cdot)$ will forward attract $B(\cdot)$. If $K(\cdot)$ is a local pullback attractor, then $K^{\varepsilon}(\cdot)$ is called a local approximate forward attractors. Then, in some cases, a local pullback attractor can illustrate the forward dynamics around itself clearly. Other recent works on forward dynamics of nonautonomous systems without compact base spaces can be found in $[6,11,13]$.

This paper is organized as follows. In Section 2 we present some basic notions and results on nonautonomous systems. In Section 3, under some general assumptions, we obtain forward attracting sets near pullback attracting sets of nonautonomous systems. We illustrate the main result with an example in Section 4.

\section{Preliminaries}

In this section we introduce some basic definitions and notions.

Let $X$ be a complete metric space with metric $d(\cdot, \cdot)$. Given $M \subset X$, we denote $\bar{M}$, int $M, \partial M$ and $M^{c}$ the closure, interior, boundary and complement of $M$ of $X$, respectively. A set $U \subset X$ is called a neighborhood of $M \subset X$, if $\bar{M} \subset$ int $U$.

The Hausdorff semidistance is defined as

$$
H_{X}(M, N)=\sup _{x \in M} d(x, N), \forall M, N \subset X .
$$

The $\varepsilon$-neighborhood of $K$ in $X$ is defined as

$$
K_{\varepsilon}=\{x \in X \mid d(x, K)<\varepsilon\} .
$$

A nonautonomous system consists of a "base flow" and a "cocycle semiflow" that is in some sense driven by the base flow.

A base flow $\left\{\phi_{t}\right\}_{t \in \mathbb{R}}$ is a group of continuous transformations from a metric space $P$ into itself such that
1) $\theta_{0}=i d_{P}$
2) $\theta_{t} \circ \theta_{s}=\theta_{t+s}$ for all $t, s \in \mathbb{R}$,
3) $\theta_{t} P=P$ for all $t \in \mathbb{R}$.

Definition 2.1. A cocycle semiflow $\phi$ on the phase space $X$ over $\theta$ is a continuous mapping $\phi: \mathbb{R}^{+} \times P \times X \rightarrow X$ satisfying

1) $\phi(0, p, x)=x$

2) $\phi(t+s, p, x)=\phi\left(t, \theta_{s} p, \phi(s, p, x)\right)$ (cocycle property).

We usually denote $\phi(t, p) x:=\phi(t, p, x)$. Then $\{\phi(t, p)\}_{t \geq 0, p \in P}$ can be viewed as a family of continuous mappings on $X$.

A nonautonomous set $B(\cdot)$ in $X$ is a family of subsets of $X$ indexed by $p \in P$,

$$
B(\cdot)=\{B(p): p \in P\} .
$$

Given a nonautonomous set $B(\cdot)$, we call $B(\cdot)$ compact in $X$ if for each $p \in P$, the set $B(p)$ is compact in $X$, and call $B(\cdot)$ forward invariant (resp. invariant) under $\phi$ if for each $p \in P$,

$$
\begin{gathered}
\phi(t, p) B(p) \subset B\left(\theta_{t} p\right), \quad t \geq 0 \\
\left(\text { resp. } \phi(t, p) B(p)=B\left(\theta_{t} p\right), \quad t \geq 0\right) .
\end{gathered}
$$

A nonautonomous set $U(\cdot)$ is called a neighborhood of $B(\cdot)$, if $\bar{B}(p) \subset$ int $U(p)$ for each $p \in P$.

A nonautonomous set $K(\cdot)$ is said to pullback (resp. forward) attracts another nonautonomous set $B(\cdot)$ under $\phi$ if for each $p \in P$,

$$
\begin{gathered}
\lim _{t \rightarrow \infty} H_{X}\left(\phi\left(t, \theta_{-t} p\right) B\left(\theta_{-t} p\right), K(p)\right)=0, \\
\left(\text { resp. } \lim _{t \rightarrow \infty} H_{X}\left(\phi(t, p) B(p), K\left(\theta_{t} p\right)\right)=0\right) .
\end{gathered}
$$

Definition 2.2. A nonautonomous set $\mathscr{A}(\cdot)$ is called a global pullback (resp. forward) attractor of $\phi$ if it is compact, invariant and pullback (resp. forward) attracts each bounded set $B \subset X$ under $\phi$.

A nonautonomous set $A(\cdot)$ is called a (local) pullback (resp. forward) attractor of $\phi$ if it is compact, invariant and pullback (resp. forward) attracts one of neighborhood $U(\cdot)$ of itself under $\phi$.

Remark 2.1. It is clear that a global attractor $\mathscr{A}(\cdot)$ will attract each neighborhood $\mathscr{U} \subset X$ of itself. However, $U(p)$ (the neighborhood of a local attractor $A(\cdot)$ ) varies in $p \in$ $P$. Therefore the definition of local attractors is a nontrivial generalization of that of global attractors.

Definition 2.3. Given a nonautonomous set $B(\cdot)$, then the omega-limit set $\omega(B)(\cdot)$ is defined to be

$$
\omega(B)(p)=\bigcap_{T \geq 0} \overline{\bigcup_{t \geq T} \phi\left(t, \theta_{-t} p\right) B\left(\theta_{-t} p\right)}, \quad p \in P .
$$

Let $\phi$ be a given cocycle semiflow on $X$ with driving system $\theta$ on base space $P$, and let $\Phi:=\{\Phi(t)\}_{t \geq 0}$ be the skew product semiflow on $Y:=P \times X$, given by

$$
\Phi(t)(p, x)=\left(\theta_{t} p, \phi(t, p) x\right), \quad t \geq 0 .
$$

Denote by $\mathbb{B} \subset Y$ the set given by

$$
\mathbb{B}=\bigcup_{p \in P}(\{p\} \times B(p))
$$

The omega-limit set $\omega(\mathbb{B})$ of $\mathbb{B}$ through $\Phi$ is defined to be

$$
\omega(\mathbb{B})=\bigcap_{T \geq 0} \overline{\bigcup_{t \geq T} \Phi(t) \mathbb{B}}
$$

We say $\mathbb{B}$ is positively invariant (resp. invariant ) under $\Phi$, if

$$
\Phi(t) \mathbb{B} \subset \mathbb{B}, \quad t \geq 0(\operatorname{resp} . \Phi(t) \mathbb{B}=\mathbb{B}, \quad t \geq 0) .
$$

It is clear that $B(\cdot)$ is forward invariant (resp. invariant) under $\phi$ if and only if $\mathbb{B}$ is positively invariant (resp. invariant) under $\Phi$.

Proposition 2.1. [8] Suppose $K(\cdot)$ and $B(\cdot)$ are two nonautonomous sets with $K(\cdot)$ attracting $B(\cdot)$, and $K(\cdot)$ is 
compact, then $\omega(B)(\cdot) \subset K(\cdot)$ is nonvoid invariant and compact, moreover $\omega(B)(\cdot)$ pullback attracts $B(\cdot)$.

As a special case of the above result, we have

Proposition 2.2. Assume $\mathbb{K}$ attracts $\mathbb{B}$ through $\Phi$ with $\mathbb{K} \subset$ $Y$ being compact. Then $\omega(\mathbb{B}) \subset \mathbb{K}$ is nonvoid invariant and compact, moreover $\omega(\mathbb{B})$ attracts $\mathbb{B}$.

\section{Local Approximate Forward Attractors of Nonautonomous Dynamical Systems}

\subsection{Equivalence Between Two Types of Attraction}

Let $(\phi, \theta)_{X, P}$ be a nonautonomous system, and let $\Phi$ be the skew-product flow associated with $\phi$. We first study the relationship between the pullback attraction of $\phi$ and the attraction of $\Phi$. More specifically, we have

Theorem 3.1. Suppose $P$ is compact. Let $K(\cdot)$ and $B(\cdot)$ be two nonautonomous sets with $K_{P}:=\overline{\bigcup_{p \in P} K(p)}$ being compact. Then $K(\cdot)$ pullback attracts $B(\cdot)$ through $\phi$ if and only if $\mathbb{K}$ attracts $\mathbb{B}$ through $\Phi$.
Remark 3.1. In the special case when $K(\cdot)$ is a global pullback attractor of $\phi$, the same results were obtained in Theorem 15.7 and Theorem 15.8 of [7].

Proof of Theorem 3.1. Necessity: By the compactness of $P$, one finds that

$$
\begin{aligned}
& \lim _{t \rightarrow \infty} H_{Y}\left(\Phi(t) \mathbb{B}, P \times K_{P}\right) \\
= & \lim _{t \rightarrow \infty} H_{X}\left(\phi(t, p) B(p), K_{P}\right) \\
\leq & \lim _{t \rightarrow \infty} \sup _{p \in P} H_{X}\left(\phi(t, p) B(p), K_{P}\right) \\
= & \lim _{t \rightarrow \infty} \sup _{p \in P} H_{X}\left(\phi\left(t, \theta_{-t} p\right) B\left(\theta_{-t} p\right), K_{P}\right)=0 .
\end{aligned}
$$

This means the compact set $P \times K_{P}$ attracts $\mathbb{B}$ through $\Phi$. Therefore the omega-limit set $\omega(\mathbb{B})$ of $\mathbb{B}$ exists and attracts $\mathbb{B}$.

In the following, we prove $\omega(\mathbb{B}) \subset \mathbb{K}$, which completes the necessity.

For this purpose, define a nonautonomous set $\tilde{B}(\cdot)$ as follows

$$
\tilde{B}(p):=\overline{\bigcup_{s \geq 0} \phi\left(s, \theta_{-s} p\right) B\left(\theta_{-s} p\right)}, \quad p \in P .
$$

It is clear that $B(\cdot) \subset \tilde{B}(\cdot)$. We first say $\tilde{B}(\cdot)$ is forward invariant. Indeed, for any $t \geq 0$ and $p \in P$,

$$
\begin{aligned}
\phi(t, p) \tilde{B}(p) & =\phi(t, p) \overline{\bigcup_{s \geq 0} \phi\left(s, \theta_{-s} p\right) B\left(\theta_{-s} p\right)} \subset \overline{\bigcup_{s \geq 0} \phi(t, p) \circ \phi\left(s, \theta_{-s} p\right) B\left(\theta_{-s} p\right)} \\
& =\overline{\bigcup_{s \geq 0} \phi\left(t+s, \theta_{-(t+s)} \circ \theta_{t} p\right) B\left(\theta_{-(t+s)} \circ \theta_{t} p\right)} \subset \overline{\bigcup_{s \geq 0} \phi\left(s, \theta_{-s} \circ \theta_{t} p\right) B\left(\theta_{-s} \circ \theta_{t} p\right)}=\tilde{B}\left(\theta_{t} p\right) .
\end{aligned}
$$

So $\tilde{B}(\cdot)$ is forward invariant, which implies the omega-limit set $\omega(\tilde{B})(\cdot)$ of $\tilde{B}(\cdot)$ is the maximal invariant set in $\tilde{B}(\cdot)$. Furthermore, for any $p \in P$,

$$
\begin{aligned}
\omega(\tilde{B})(p) & =\bigcap_{\tau \geq 0} \overline{\bigcup_{t \geq \tau} \phi\left(t, \theta_{-t} p\right) \tilde{B}\left(\theta_{-t} p\right)}=\bigcap_{\tau \geq 0} \overline{\bigcup_{t \geq \tau} \phi\left(t, \theta_{-t} p\right) \overline{\bigcup_{s \geq 0} \phi\left(s, \theta_{-(s+t)} p\right) B\left(\theta_{-(s+t)} p\right)}} \\
& =\bigcap_{\tau \geq 0} \overline{\bigcup_{t \geq \tau} \phi\left(t, \theta_{-t} p\right) \bigcup_{s \geq 0} \phi\left(s, \theta_{-(s+t)} p\right) B\left(\theta_{-(s+t)} p\right)}=\bigcap_{\tau \geq 0} \overline{\bigcup_{t \geq \tau} \phi\left(t, \theta_{-t} p\right) \circ \phi\left(s, \theta_{-(s+t)} p\right) B\left(\theta_{-(s+t)} p\right)} \\
& =\bigcap_{\tau \geq 0} \overline{\bigcup_{t \geq \tau} \phi\left(t+s, \theta_{-(s+t)} p\right) B\left(\theta_{-(s+t)} p\right)}=\bigcap_{\tau \geq 0} \overline{\bigcup_{t \geq \tau} \phi\left(t, \theta_{-t} p\right) B\left(\theta_{-t} p\right)}=\omega(B)(p),
\end{aligned}
$$

where the third " $=$ " holds since for each fixed $t \geq 0$ and $p \in P, \phi\left(t, \theta_{-t} p\right)$ is a continuous map on $X$. It follows that $\omega(B)(\cdot)$ is the maximal forward invariant set in $\tilde{B}(\cdot)$. Therefore $\mathbb{C}:=\bigcup_{p \in P}(\{p\} \times \omega(B)(p))$ is the maximal invariant set in $\tilde{\mathbb{B}}:=\bigcup_{p \in P}(\{p\} \times \tilde{B}(p))$. By the forward invariance of $\tilde{B}(\cdot)$,

$$
\begin{aligned}
\phi(t) \tilde{\mathbb{B}} & =\phi(t) \bigcup_{p \in P}(\{p\} \times \tilde{B}(p)) \subset \bigcup_{p \in P} \phi(t)(\{p\} \times \tilde{B}(p)) \\
& =\bigcup_{p \in P}\left(\left\{\theta_{t} p\right\} \times \phi(t, p) \tilde{B}(p)\right) \subset(\text { by }(1)) \subset \bigcup_{p \in P}\left(\left\{\theta_{t} p\right\} \times \tilde{B}\left(\theta_{t} p\right)\right) \\
& =\tilde{\mathbb{B}}, \quad t \geq 0
\end{aligned}
$$


i.e. $\quad \tilde{\mathbb{B}}$ is positively invariant under $\phi$. Then $\omega(\tilde{\mathbb{B}})$ is the maximal invariant set in $\tilde{\mathbb{B}}$. Recall that $\mathbb{C}$ is also the maximal invariant set in $\tilde{\mathbb{B}}$, we have

$$
\omega(\mathbb{B}) \subset \omega(\tilde{\mathbb{B}})=\mathbb{C} .
$$

Finally, by the assumption that $K(\cdot)$ attracts $B(\cdot)$, one knows that $\omega(B)(\cdot) \subset K(\cdot)$, and thus $\mathbb{C} \subset \mathbb{K}$, which shows

$$
\omega(\mathbb{B}) \subset \mathbb{K} .
$$

Sufficiency: In a very similar way as above, we can prove the sufficiency.

By the compactness of $P$,

$$
\begin{aligned}
& \left.\lim _{t \rightarrow \infty} H_{X}\left(\phi\left(t, \theta_{-t} p\right) B\left(\theta_{-t} p\right), K_{P}\right)\right] \\
\leq & \lim _{t \rightarrow \infty} \sup _{p \in P} H_{X}\left(\phi(t, p) B(p), K_{P}\right) \\
= & \lim _{t \rightarrow \infty} \sup _{p \in P} H_{Y}\left(\Phi(t) \mathbb{B}, P \times K_{P}\right) \\
= & \lim _{t \rightarrow \infty} H_{Y}\left(\Phi(t) \mathbb{B}, P \times K_{P}\right)=0,
\end{aligned}
$$

which implies $\omega(B)(\cdot)$ exists and pullback attracts $B(\cdot)$.

To complete the proof, it suffices to show $\omega(B)(\cdot) \subset K(\cdot)$. We first define a set

$$
\hat{\mathbb{B}}=\overline{\bigcup_{s \geq 0} \Phi(s) \mathbb{B}} .
$$

Then $\hat{\mathbb{B}}$ is positively invariant and

$$
\omega(\hat{\mathbb{B}})=\omega(\mathbb{B}) .
$$

This implies that $\Omega(\mathbb{B})$ is the maximal invariant set in $\hat{\mathbb{B}}$. Write $\omega(\mathbb{B}):=\bigcup_{p \in P}\{p\} \times C(p)$, then $C(\cdot)$ is the maximal invariant set in $\hat{B}(\cdot)$, where $\hat{B}(\cdot)$ is the set defined by $\hat{\mathbb{B}}:=$ $\bigcup_{p \in P}\{p\} \times \hat{B}(p)$. By the positive invariance of $\hat{\mathbb{B}}$, one also knows that $\hat{B}(\cdot)$ is forward invariant. This implies $\Omega_{\hat{B}}(\cdot)$ is the maximal invariant set in $\hat{B}(\cdot)$. We then have that $\omega(B)(\cdot) \subset$ $\omega(\hat{B})(\cdot)=C(\cdot)$. We learn from the condition $\omega(\mathbb{B}) \subset \mathbb{K}$ that $C(\cdot) \subset K(\cdot)$. In summary, $\omega(B)(\cdot) \subset K(\cdot)$, which completes the sufficiency.

\subsection{Local Approximate Forward Attractors}

Definition 3.1. Let $K(\cdot)$ be a nonautonomous set of $X$. We call $K^{\varepsilon}(\cdot)$ the $\varepsilon$-extended neighborhood of $K(\cdot)$ if the set $\mathbb{K}_{\varepsilon}:=\bigcup_{p \in P}\left(\{p\} \times K^{\varepsilon}(p)\right)$ is the $\varepsilon$-neighborhood of $\mathbb{K}=\bigcup_{p \in P}(\{p\} \times K(p))$ in the space $Y$.

Then, under some suitable assumptions, we can construct local approximate forward attractors around pullback attractors. In fact, we get a stronger result as follows.

Theorem 3.2. Let $\phi$ be a cocycle semiflow on a Banach space $X$ with driving system $\theta$ on a compact base space $P$. Let $K(\cdot)$ be a set such that $K_{P}$ is compact. If $K(\cdot)$ has pullback attraction through $\phi$, then for any $\varepsilon>0$, the $\varepsilon$-extended neighborhood $K^{\varepsilon}(\cdot)$ of $K(\cdot)$ is an approximate forward attracting set associated to $K(\cdot)$, namely, if $K(\cdot)$ pullback attracts a nonautonomous set $B(\cdot)$, then $K^{\varepsilon}(\cdot)$ forward attracts $B(\cdot)$ uniformly on $p \in P$, i.e.

$$
\lim _{t \rightarrow \infty} \sup _{p \in P} H_{X}\left(\phi(t, p) B(p), K^{\varepsilon}\left(\theta_{t} p\right)\right)=0 .
$$

Proof. In the first part of the proof, we employ many of the techniques developed in [18, Theorem 3.1], where they were used to construct global pullback attracting sets for cocycle semiflows.

We know from Theorem 3.1 that $\mathbb{K}$ attracts $\mathbb{B}$, i.e. for any $\delta>0$ (we can assume $\delta<\varepsilon$ ) there is a $T>0$ (being independent of $p \in P$ ) so large that

$$
H_{Y}(\Phi(t) \mathbb{B}, \mathbb{K})<\delta / 2, \quad t \geq T .
$$

It follows that for any $u=\left(p, u^{\prime}\right) \in \mathbb{B}$, there is a $v \in \mathbb{K}$ such that

$$
d_{Y}\left(\left(\theta_{t} p, \phi(t, p) u^{\prime}\right), v\right) \leq \delta, \quad t \geq T
$$

Denote $q:=\theta_{t} p$. Then (2) can be written as

$$
d_{Y}\left(\left(q, \phi\left(t, \theta_{-t} q\right) u^{\prime}\right), v\right) \leq \delta, \quad t \geq T .
$$

For each $q \in P$, we divide $\mathbb{K}$ in two parts,

$$
\mathbb{K}:=\mathbb{K}_{q}[\varepsilon] \bigcup \mathbb{K}_{q}^{c}[\varepsilon]
$$

where

$$
\mathbb{K}_{q}[\varepsilon]:=\bigcup_{d(p, q)<\varepsilon}(\{p\} \times K(p))
$$

and

$$
\mathbb{K}_{q}^{c}[\varepsilon]:=\bigcup_{d(p, q) \geq \varepsilon}(\{p\} \times K(p)) .
$$

If $v \in \mathbb{K}_{q}^{c}[\varepsilon]$, then for $t \geq T$,

$$
d_{Y}\left(\left(q, \phi\left(t, \theta_{-t} q\right) u^{\prime}\right), v\right)>d(q, p) \geq \varepsilon>\delta,
$$

which contradicts to (3). So we necessarily have $v \in \mathbb{K}_{q}[\varepsilon]$. Write $v:=\left(q^{\prime}, v^{\prime}\right)$ and define a nonautonomous set $K^{[\varepsilon]}(\cdot)$ by

$$
K^{[\varepsilon]}(q)=\bigcup_{d(p, q)<\varepsilon} K(p), \quad q \in P .
$$

Since $d_{P}\left(q^{\prime}, q\right)<\varepsilon$, we have $v^{\prime} \in K^{[\varepsilon]}(q)$.

It follows that

$$
\begin{aligned}
& d_{X}\left(\phi\left(t, \theta_{-t} q\right) u^{\prime}, K^{[\varepsilon]}(q)\right) \leq d_{X}\left(\phi\left(t, \theta_{-t} q\right) u^{\prime}, v^{\prime}\right) \\
& \quad \leq d_{X}\left(\left(q, \phi\left(t, \theta_{-t} q\right) u^{\prime}\right), v\right) \leq \delta, \quad t \geq T .
\end{aligned}
$$

Rewrite $q$ as $\theta_{t} p$, we have

$$
d_{X}\left(\phi(t, p) u^{\prime}, K^{[\varepsilon]}\left(\theta_{t} p\right)\right) \leq \delta<\varepsilon, \quad t \geq T .
$$


Since $u^{\prime} \in B(p)$ is arbitrary, we can conclude that

$$
\mathrm{H}_{\mathrm{X}}\left(\phi(\mathrm{t}, \mathrm{p}) \mathrm{B}(\mathrm{p}), \mathrm{K}^{[\varepsilon]}\left(\theta_{\mathrm{t}} \mathrm{p}\right)\right) \leq \delta, \quad \text { for all } \mathrm{t} \geq \mathrm{T} .
$$

By the arbitrariness of $\delta$ and the independence of $T$ on $p \in P$, one knows $K^{[\varepsilon]}(\cdot)$ forward attracts $B(\cdot)$ uniformly on $p \in P$.

In the rest, we prove $K^{[\varepsilon]}(\cdot) \subset K^{\varepsilon}(\cdot)$, which completes the proof of the theorem.

Let $p \in P$ be fixed. We first claim that for any $q \in P$ with $d_{P}(q, p)<\varepsilon$, it holds that

$$
K(q) \subset K^{\varepsilon}(p)
$$

Indeed, for any $q \in P$ with $d_{P}(q, p)<\varepsilon$ and $x \in K(q)$, we have

$$
d_{Y}((p, x),(q, x))=d_{P}(p, q)=d_{P}(q, p)<\varepsilon .
$$

Since $(q, x) \in \mathbb{K}$, one knows that $(p, x) \in \mathbb{K}_{\varepsilon}$, that is, $x \in K^{\varepsilon}(p)$, which proves the assertion. We then immediately get from the definition

$$
K^{[\varepsilon]}(p)=\bigcup_{d(q, p)<\varepsilon} K(q), \quad p \in P
$$

that

$$
K^{[\varepsilon]}(p) \subset K^{\varepsilon}(p)
$$

\section{An Example}

We study the forward dynimics of the nonautonomous scalar model

$$
\dot{x}=x-h(t) x^{2},
$$

where $h \in C_{b}(\mathbb{R}, \mathbb{R})$, the set of bounded continuous functions from $\mathbb{R}$ to $\mathbb{R}$, and $h(t) \geq c>0, t \in \mathbb{R}$ for some constant $c$. We can obtain the explicit solution

$$
x\left(t, s ; x_{0}\right)=\frac{e^{t}}{x_{0}^{-1} e^{s}+\int_{s}^{t} e^{r} h(r) d r},
$$

with $x(s)=x_{0} \neq 0$.

The unique solution can generate a process $S(\cdot, \cdot):=$ $\{S(t, s)\}_{t \geq s}$ on $\mathbb{R}$ by $S(t, s) x_{0}=x\left(t, s ; x_{0}\right)$.

It is clear that 0 is an equilibrium. For $x_{0}>0$ fixed, letting $s \rightarrow-\infty$ in (5) yields

$$
x^{*}(t)=\frac{e^{t}}{\int_{-\infty}^{t} e^{r} h(r) d r} .
$$

This shows that $x^{*}(t)$ is a local pullback attractor for the process $S(\cdot, \cdot)$ in $(0, \infty)$.

In order to study the forward dynamics in $(0, \infty)$, we work in the framework of cocycle semiflows and require additional assumption on $h$. Define the hull of $h(t)$ as follows

$$
\mathcal{H}:=\mathcal{H}[h(\cdot)]=\overline{\{h(\tau+\cdot): \tau \in \mathbb{R}\}} C_{b}(\mathbb{R}, \mathbb{R}) .
$$

In application, $h(\cdot)$ is often taken as a periodic function, quasiperiodic function, almost periodic function, local almost periodic function $[3,14]$ or uniformly almost automorphic function [19], and the metric on $C_{b}(\mathbb{R}, \mathbb{R})$ is taken as uniform convergence on compact subintervals. In this case, the hull $\mathcal{H}$ is a compact metric space. A simple example is that $h(t)=\sin t, t \in \mathbb{R}$.

Accordingly, the translation group $\theta$ on $\mathcal{H}$ is given by

$$
\theta_{\tau} p(\cdot)=p(\tau+\cdot), \quad t \in \mathbb{R}, p \in \mathcal{H} .
$$

Instead of (4), we will consider the more general cocycle system:

$$
\dot{x}=x-p(t) x^{2}, \quad p \in \mathcal{H} .
$$

Then the unique solution $x\left(t, 0 ; x_{0}, p\right)$ will generate a cocycle semiflow $\phi$ on $\mathbb{R}$ with driving system $\theta$ on a base space $\mathcal{H}$, which is given by $\phi(t, p) x_{0}=x\left(t, 0 ; x_{0}, p\right)$.

Leaning from $(6)$, we can define a local pullback attractor $x_{*}(\cdot)$ for $\phi$ by

$$
x_{*}(p)=\frac{1}{\int_{-\infty}^{0} e^{r} p(r) d r} .
$$

It is worth noting that

$$
x_{*}\left(\theta_{t} h\right)=x^{*}(t)
$$

is the full solution of (4) given in (6).

Since $h(t) \geq c>0, t \in \mathbb{R}$, we have

$$
\sup _{p \in P} x_{*}(p) \leq 1 / c .
$$

Then by Theorem 3.2, we have

Theorem 4.1. Suppose $h$ is a function such that $\mathcal{H}$ is compact and $(7)$ holds. Then for any $\varepsilon>0$, the $\varepsilon$-extended neighborhood $x_{*}^{\varepsilon}(\cdot)$ of $x_{*}(\cdot)$ has forward attraction. It will forward attracts every bounded set $B$ in $(0, \infty)$ uniformly on $p \in P$, i.e.

$$
\lim _{t \rightarrow \infty} \sup _{p \in P} H_{\mathbb{R}}\left(\phi(t, p) B, x_{*}^{\varepsilon}\left(\theta_{t} p\right)\right)=0 .
$$

In particular for the equation (4), there is a neighborhood $\mathcal{O}(t)$ of the full solution $x^{*}(t)$ such that $\mathcal{O}(t)$ forward attracts every solution $x\left(t, s ; x_{0}\right)$ with $x_{0}>0$, that is,

$$
\lim _{t \rightarrow \infty} H_{\mathbb{R}}\left(x\left(t, s ; x_{0}\right), \mathcal{O}(t)\right)=0 .
$$

\section{Conclusion}

Our goal of this paper is to study the local forward dynamics of nonautonomous dynamical systems, which can be seen as a generalisation of our previous paper [10] where the global forward dynamics was considered. However, the results here is not trivial since we have to apple different methods. It is worth noting that the compactness assumption on the base space $P$ is very necessary. It is still hard to study the forward dynamics 
of a nonautonomous dynamical system without a compact base space.

\section{Acknowledgements}

The authors gratefully acknowledge the support of the Foundation Research Funds for the Central Universities under Grant No. 3122019145.

\section{References}

[1] E. Aragao-Costa, T. Caraballo, A. Carvalho and J.A. Langa, Non-autonomous Morse-decomposition and Lyapunov functions for gradient-like processes. Transactions of the American Mathematical Society, 365 (10), (2013), 5277-5312.

[2] D. Cheban, P. Kloeden and B. Schmalfuss, The relation between pullback and global attractors for nonautonomous dynamical systems, Nonlinear Dyn. Syst. Theory 2 (2002), 125-144.

[3] V. V. Chepyzhov and M. I. Vishik, Attractors of Equations of Mathematical Physics, Amer. Math. Soc., Providence, RI, 2002.

[4] V. V. Chepyzhov and M. I. Vishik, Attractors of nonautonmous dynamical systems and their dimension, J. Math. Pures Appl., 73, (1994), 279-333.

[5] T. Caraballo, J. C. Jara, J. A. Langa and Z.X. Liu, Morse decomposition of attractors for non-autonomous dynamical systems, Advanced Nonlinear Studies, 13 (2), (2013), 309-329.

[6] T. Caraballo, J. A. Langa and R. Obaya, Pullback, forward and chaotic dynamics in 1D non-autonomous linear-dissipative equations, Nonlinearity 30. 1 (2017), 274-299.

[7] A. N. Carvalho, J. A. Langa and J. C. Robinson, Attractors for Infinite-Dimensional Non autonomous Dynamical Systems, Appl. Math. Sci. 182, 2013.

[8] H. Crauel and F. Flandoli, Attractors for random dynamical systems, Probab. Theory Related Fields 100, (1994) 365-393.
[9] H. Crauel, A. Debussche and F. Flandoli, Random Attractors, J. Dynam. Differential Equations 9 (1997), 307-341.

[10] X. W. Ju, D. S. Li, C. Q. Li and A. L. Qi, Approximate forward attractors of non-autonomous dynamical systems, Chinese Annals of Mathematics, Series B, 2019, 40 (4), 541-554.

[11] P. E. Kloeden, Asymptotic invariance and the discretisation of non-autonomous forward attracting sets, J. Comput. Dynamics, 3 (2016), 179-189.

[12] P. E. Kloeden and T. Lorenz, Construction of nonautonomous forward attractors, Proc. Amer. Math. Soc., (2016), 259-268.

[13] H. Y. Cui and P. E. Kloeden, Invariant forward attractors of non-autonomous random dynamical systems, Journal of Differential Equations 265.12 (2018), 6166-6186.

[14] D. S. Li and J. Q. Duan, Structure of the set of bounded solutions for a class of nonautonomous secondorder differential equations, J. Differential Equations 246 (2009), 1754-1773.

[15] A. N. Carvalho, J. A. Langa, J. C. Robinson and A. Suárez, Characterization of nonautonomous attractors of a perturbed gradient system, J. Differential Equations 236 (2007), 570-603.

[16] M. Rasmussen, Morse decompositions of nonautonomous dynamical systems, Trans. Amer. Math. Soc. 359 (2007), no. 10, 5091-5115.

[17] M. I. Vishik, Asymptotic Behavior of Solutions of Evlutionary Equations, Cambridge University Press, Cambriage, England, 1992.

[18] Y. J. Wang, D. S. Li, P. E. Kloeden, On the asymptotically behavior of non-autonomous dynamical systems, Nonlinear Anal., 59 (2004), 35-53.

[19] Y. Yi and W. Shen, Almost automorphic and almost periodic dynamics in skew-product semiflows, Mem. Amer. Math. Soc., 136 (651), 647-647. 